\title{
Differences in echocardiography, blood pressure, stroke volume, maximal power and profile of genes related to cardiac hypertrophy in elite road cyclists
}

\author{
Grażyna Janikowska ${ }^{1, A-F}$, Aleksandra Żebrowska ${ }^{2, A-F}$, Aleksandra Kochańska-Dziurowicz ${ }^{3,4, B, C, E, F}$, Urszula Mazurek ${ }^{5, C, F}$ \\ ${ }^{1}$ Department of Analytical Chemistry, Medical University of Silesia, Katowice, Poland \\ 2 Department of Physiology, The Jerzy Kukuczka Academy of Physical Education, Katowice, Poland \\ ${ }^{3}$ Department of Isotope Diagnostic and Radiopharmacy, Medical University of Silesia, Katowice, Poland \\ ${ }^{4}$ Department of Health Care, Silesian Medical College, Katowice, Poland \\ ${ }^{5}$ Department of Molecular Biology, Medical University of Silesia, Katowice, Poland \\ A - research concept and design; $\mathrm{B}$ - collection and/or assembly of data; $\mathrm{C}$ - data analysis and interpretation; \\ $D$ - writing the article; $E$ - critical revision of the article; $F$ - final approval of article
}

Address for correspondence

Grażyna Janikowska

E-mail: gjanikowska@sum.edu.pl

Funding sources

The study was funded by the grants from the Medical University of Silesia (KNW-1023/K/4/0/2/14/15; KNW-1-024/N/5/0).

Conflict of interest None declared

Received on November 28, 2015

Revised on March 8, 2016

Accepted on May 5, 2016

DOI

10.17219/acem/63031

Copyright

Copyright by Author(s)

This is an article distributed under the terms of the

Creative Commons Attribution Non-Commercial License

(http://creativecommons.org/licenses/by-nc-nd/4.0/)

\section{Abstract}

Background. Regular and moderate exercise is beneficial for improving the efficiency of the heart, but high-intensity physical activity may result in cardiac changes.

Objectives. This study focuses on the identification of the differences in echocardiography and blood variables before exercise, as well as the genes associated with cardiac hypertrophy at rest and in response to graded exercise test.

Material and methods. The study group was made up of 28 road cyclists. Echocardiographic parameters and blood pressure were measured before exercise tests $(N=28)$. Blood samples were collected at rest, at maximal exercise intensity in a graded bicycle test and after 15 min of recovery; afterwards, blood morphology was estimated and RNA was isolated. Analysis of the expression profile of genes was performed for randomly selected road cyclists using the microarray method.

Results. Echocardiographic results and blood parameters divided cyclists into two groups: with and without left ventricular hypertrophy $(N=14)$. Differences in the structure and function of the left ventricle cyclists with a similar level of training were observed $(p<0.05)$. Diastolic blood pressure and resting heart rate were significantly lower in subjects with left ventricular hypertrophy $(p<0.05)$. The myosin light chain 9 and interleukin-6 signal transducer gene expression were differentially regulated in cyclists with left ventricular hypertrophy compared to athletes with normal heart dimensions in response to intensive exercise.

Conclusions. We have found differences in echocardiography parameters, blood pressure, stroke volume and maximal power in the cyclists examined. These studies indicate the benefits of the recommended echocardiography measurements for professional endurance-athletes. The graded exercise altered the myosin light chain 9 and interleukin-6 signal transducer gene expression in the peripheral blood of road cyclists has also been found.

Key words: gene expression, exercise, echocardiography, left ventricular hypertrophy, road cyclists 
It is well-known that regular and moderate exercise is beneficial for improving the efficiency of the heart. Among others benefits, it reduces the risk of atherosclerosis and improves exercise tolerance in patients with coronary pain and heart failure. ${ }^{1}$ High-intensity physical activity may result in cardiac dysfunction and increased incidents of sudden death in athletes. These observations can be found in studies demonstrating that extreme physical load can lead to the transient reduction of the left ventricular function and increases the incidence of arrhythmogenic right ventricular cardiomyopathy. ${ }^{2,3}$ That is why the world's sporting and cardiology organizations recommend that medical examinations be performed on competitive endurance athletes for the early detection of possible pathologies. ${ }^{4,5}$ Strenuous exercise, such as road cycling, may result in compensatory myocardial hypertrophy, commonly known as athlete's heart. ${ }^{6}$ The main morphologic characteristics are left ventricular hypertrophy $(\mathrm{LVH})$, an increase in the coronary reserve and improvement of left ventricular myocardial contractility. ${ }^{6,7}$ The primary stimulus for cardiac hypertrophy is mechanical stress, which induces a growth response in the overloaded myocardium. ${ }^{7}$ The possible mechanisms of cardiac hypertrophy remain controversial. Several researchers have highlighted the importance of hormonal regulation or an adrenergic nervous system or other factors. ${ }^{8,9}$ It has also been suggested that mechanical loading regulates intracellular signals for the gene expression associated with cardiac hypertrophy without participation of humoral or neural factors. Although alterations in the expression of genes implicated in cardiac hypertrophy have been extensively studied in experimental models ${ }^{10}$ and clinical studies ${ }^{1,11}$ little is known regarding the signaling mechanisms that lead to the gene expression in the peripheral blood of trained cyclists. Literature reviews indicate that all structural and functional changes, which represent physiological adaptation to regular training, increase the functional capacity of the cardiovascular system at rest and during exercise. ${ }^{9-11}$ It should be taken into consideration that several mechanisms may be involved in exercise-related cardiac fatigue, including changes in preload conditions, myocardial stunning, $\beta_{1}$-receptor desensitization, the impaired release of cardiac biomarkers and altered autonomic regulation..$^{9-12}$ Intriguingly, a remarkably different gene expression pattern was noted between adaptive and maladaptive cardiac hypertrophy. ${ }^{13,14}$ Little is known regarding the gene expression which is considered to be related to left ventricular hypertrophy in endurance-trained athletes. Moreover, it has not been clearly defined whether the genes' expression changes in response to maximal physical exertion and/or post-exercise recovery.

Therefore, the goal of this study was to investigate the differences in echocardiographic and other variables before exercise, as well as in the expression profile of genes associated with cardiac hypertrophy at rest and in re- sponse to maximal physical exertion and post-exercise recovery in professional road cyclists.

\section{Material and methods}

\section{Subjects and study protocol}

Twenty-eight male Polish elite road cyclists gave their informed written consent to participate in this study. All subjects were competitive cyclists with the same training status of LVH and non-LVH group $(9.9 \pm 5.1$ and $9.3 \pm 4.3$ years, respectively). The training status of the subjects is expressed as maximal oxygen consumption $\left(\mathrm{VO}_{2 \max }\right)$, the highest value was $71 \mathrm{~mL} / \mathrm{kg} / \mathrm{min}$, and the mean individual monthly training volume was $655 \pm 53 \mathrm{~km}$. They participated in the study during the pre-season period and underwent medical evaluations including clinical history, physical examinations, echocardiography and blood parameters. The study was approved by the local ethics committee and conformed to the standard set by the declaration of Helsinki.

Pre- and post-exercise data collections were carried out in an exercise physiology laboratory in the morning hours between 08.00 and 11.00. None of the subjects had a history of cardiovascular, pulmonary or metabolic diseases. Three days prior to the study all participants were put on a mixed, isocaloric diet $(2899 \pm 1100 \mathrm{kcal} /$ day $)$ consisting of carbohydrates, proteins and fats. The athletes were asked to refrain from food, juices and caffeine for at least 8 hours and avoid strenuous exercise for 24 hours prior to testing.

\section{Echocardiographic data}

All examinations were performed using the HewlettPackard Image Point HX ultrasound system with standard imaging transducers. All analyses were made and/ or supervised by the same cardiologist. Left ventricular end-diastolic dimensions (LVEDD) were measured at the onset of the QRS complex. LV volumes were derived according to the modified Simpson's method. The left ventricular ejection fraction (LVEF) was calculated in the standard fashion from LV end-diastolic and end-systolic volume.

The intra-observer coefficients of variation were $2.5 \%$ for the measurements of LV diameters, and 3.6\% for measurements of LVEF. The left ventricular systolic function was determined by measurements of LVEF and stroke volume (SV). LV muscle mass (LVM) was derived according to Devereux. ${ }^{15}$ LV mass $=1.04$ [(LVEDD + IVSDD + LVPWT) - (LVEDD)], where LVEDD was the left ventricular end-diastolic internal diameter, IVSDD was the intraventricular septum thickness during diastole, and LVPWT was the left ventricular posterior wall thickness. ${ }^{3}$ Left ventricular mass was indexed to body surface 
area (BSA). Left ventricular hypertrophy was identified when left ventricular mass index (LVM/BSA) was greater than $115 \mathrm{~g} / \mathrm{m}^{2}$ in men. ${ }^{4}$

\section{Maximal graded exercise test}

Before each subject performed a maximal graded exercise test using a cycle ergometer (F-Lode Excalibur, Groningen, Netherlands), blood pressure and heart rate were measured with a manual sphygmomanometer. The exercise started with unloaded cycling for $5 \mathrm{~min}$, and then increased by $40 \mathrm{~W}$ every $3 \mathrm{~min}$, up to the maximal exercise intensity. The maximal oxygen uptake $\left(\mathrm{VO}_{2 \mathrm{max}}\right)$ was achieved if two of the following criteria occurred: a plateau in oxygen uptake $\left(\mathrm{VO}_{2}\right)$ with increasing work rate; respiratory quotient greater than 1.15; a heart rate (HR) within 10 beats of age predicted maximum (220 - age); or volitional fatigue. Oxygen uptake was measured continuously from the $6^{\text {th }}$ minute prior to exercise and throughout each stage of the exercise protocol using the Oxycon Apparatus (Jaeger, Germany).

\section{Biochemical data}

Following the exercise testing procedures, blood samples were taken using a peripheral catheter inserted into the antecubital vein. Venous blood samples were collected in a sitting position: 15 min before exercise $\left(\mathrm{T}_{\text {rest }}\right)$, at maximal exercise intensity $\left(\mathrm{T}_{\max }\right)$, and after $15 \mathrm{~min}$ of recovery $\left(\mathrm{T}_{15}\right)$. Morphology variables in the whole blood were measured with the ABX MICROS 60 (HORIBA ABX SAS) analyzer, using standard methods and reagents.

\section{Microarray analysis}

Immediately after the whole blood sample collection, leukocytes were separated using the standard method. Total RNA was isolated from leukocytes according to the manufacturer's protocol with a ready-to-use TRIZOL ${ }^{\circledR}$ Reagent (Invitrogen Life Technologies, USA). The obtained RNA was purified using RNeasy Mini kit columns with DNase I from Qiagen (Germany). The amount of the obtained RNA and its purity was checked using a GeneQuant II spectrophotometric calculator (Pharmacia Biotech, UK), at wavelengths of 1 230/260/280/320 nm. To check the specificity of gene expression changes in cyclists with left ventricular hypertrophy, the microarray data from randomly selected cyclists in both groups was analyzed. The isolated RNA samples were used for cDNA and biotin-labeled cRNA synthesis, which was then fragmented and hybridized with Affymetrix Human Genome U133A expression microarray in accordance with the Gene Expression Analysis Technical Manual (Affymetrix, USA). Fluorescence signals (FS) of the probe set were scanned by the GeneArray Scanner 3000 7G (Affymetrix, USA).

\section{Statistical analysis}

The obtained results were analyzed using the Statistica for Windows, v. 12 (StatSoft Inc.). The Shapiro-Wilks W test was used to confirm normal distribution. Values are presented as mean \pm standard deviation (SD). Student's t-test was used for comparison between groups (LVH and non-LVH). Using the Affymetrix NetAffx database, 56 mRNAs were selected, which corresponded to cardiac hypertrophy-related genes. The obtained fluorescence signals (FS) were normalized by the RMA Express $\left(\log _{2}\right.$ FS) using Matlab software. ANOVA with the Tukey post hoc tests were used to analyze changes in MYL9 and IL6ST over time $\left(\mathrm{T}_{\text {rest }}, \mathrm{T}_{\max }, \mathrm{T}_{15}\right)$. According to the standard method for analyzing the microarray data of gene expression, the results were calculated as a ratio of the normalized FS for the LVH and non-LVH, i.e. as mRNA fold change value (FC). ${ }^{16}$ Statistical significance was set at $\mathrm{p} \leq 0.05$. The Figure was performed using Microsoft Office Excel 2007 (Microsoft Co.).

\section{Results}

Road cyclists characteristics are shown in Table 1. Echocardiography measurements indicated that 14 male athletes (the LVH group) left ventricular hypertrophy was diagnosed. No symptoms of cardiovascular complications were observed in the athletes with and without LVH (Table 1).

We did not find any differences in age, body mass, body mass index, and body surface area as well as the percentage of body fat between athletes without and with LVH (Table 1). The training status of the non-LVH and LVH subjects are expressed as maximal oxygen consumption and aerobic power peak and are presented in Table 1 . No significant difference was observed in $\mathrm{VO}_{2 \max }$ between non-LVH and LVH individuals. However, peak aerobic power was significantly higher in LVH than in non-LVH subjects (Table 1). Regardless of anthropometric characteristics, significant differences in echocardiographic parameters were observed between our cyclists.

The results of the echocardiography examinations of the athletes (e.g. left ventricular mass index, intraventricular septum thickness during diastole, left ventricular posterior wall thickness) and function (stroke volume) were significantly higher compared to normal heart dimensions clearly indicating adaptive heart hypertrophy in the LVH group (Table 1).

Resting heart rate (HR) was significantly lower in the LVH group compared to the non-LVH participants. Systolic blood pressure did not differ between the groups; however, diastolic blood pressure was significantly lower in LVH subjects. No significant difference was observed regarding blood morphology variables (Table 1). Maximal oxygen uptake $\left(\mathrm{VO}_{2 \max }\right)$ was similar for all subjects; 
however, LVH athletes demonstrated significantly higher power output compared to non-LVH athletes.

The most interesting finding was that the gene expression related to cardiac hypertrophy was regulated differently before exercise and/or post exercise and during recovery. The analysis of global gene expression at rest, maximal exercise intensity and after $15 \mathrm{~min}$ of recovery revealed that 14 of the 37 genes', associated with cardiac hypertrophy, profile had changed in at least one period of the exercise test. It is interesting to note that there were significant differences only in the Myosin Light chain 9 (MYL9) and Interleukin 6 Signal Transducer (IL6ST) gene expression when comparing the cyclists with LVH and non-LVH in $\mathrm{T}_{\max }(\mathrm{FC}>1.1 ; \mathrm{p}<0.05 ;$ Fig. 1). The statistically significant differences for MYL9 were observed in $\mathrm{T}_{\max }$ and $\mathrm{T}_{15}$ in $\mathrm{LVH}$ cyclists when compared to non-LVH and up-regulated FC values were 1.56 and 1.4 , respectively $(\mathrm{p}<0.03)$. IL6ST was down-regulated in all phases of the experiment and significant decreased expression only at maximal exercise intensity $\left(\mathrm{T}_{\max }\right)$ of the LVH compared to non-LVH subjects was found ( $\mathrm{FC}=-1.17 ; \mathrm{p}=0.03$; Fig. 1). We did not find any significant differences in the expression of other cardiac hypertrophy-related genes in LVH cyclists, either at rest or in response to physical exercise.

\section{Discussion}

Among the twenty-eight cyclists examined two groups were selected, non-LVH $\left(\leq 115 \mathrm{~g} / \mathrm{m}^{2}\right)$ and LVH $(>115 \mathrm{~g} /$ $\mathrm{m}^{2}$ ) based on LVMI, where characteristics differed concerning physical capacity, and the function of the left ventricular as well as diastolic pressure and heart rate (Table 1). It is well established in sport physiology that endurance training may improve exercise capacity and cardiac function at rest and during exercise. ${ }^{5,17}$ Echocardiographic examinations of all cyclists showed that LVH subjects had an increase in LVMI ( $<<0.001)$, as well as, higher LVPWT $(\mathrm{p}<0.05)$ and IVSDD $(\mathrm{p}<0.05)$. Similar differences were observed in the mass of the left ventricular in other endurance athletes. ${ }^{18}$ This is contrary to an Israeli study where cyclists were found to have lower values of LVEDD, even in non-LVH, and 5\% of those examined were similar to the LVH group in our study. ${ }^{19} \mathrm{Cardi}-$ ac function at rest, as determined by higher SV and lower resting $H R$, revealed better left ventricular adaptation to endurance training (Table 1). The cyclists did not participate in the echocardiographic study before beginning their sporting activity. Therefore, we can only speculate about the possible impact of endurance training on the cardiac structure and function. The cardiovascular effects of endurance training include decreased heart rate, increased stroke volume of the heart and increased cardiac output as well as total mitochondrial volume in the slow twitch muscle fibers. All these factors have been shown to have beneficial effects on higher efficiency in
Table 1. Characteristic variables of the examined cyclists measured before exercise test

\begin{tabular}{|c|c|c|c|}
\hline \multirow{2}{*}{ Variables } & \multicolumn{2}{|c|}{ Subjects } & \multirow{2}{*}{$p$-value } \\
\hline & $\begin{array}{c}\text { non-LVH } \\
(\mathrm{n}=14)\end{array}$ & $\begin{array}{c}\text { LVH } \\
(n=14)\end{array}$ & \\
\hline Age [years] & $25.1 \pm 8.8$ & $26.2 \pm 6.9$ & ns \\
\hline Body mass [kg] & $72.0 \pm 6.9$ & $72.0 \pm 7.1$ & ns \\
\hline BMI $\left[\mathrm{kg} / \mathrm{m}^{2}\right]$ & $22.7 \pm 2.2$ & $22.4 \pm 1.9$ & ns \\
\hline $\mathrm{BSA}\left[\mathrm{m}^{2}\right]$ & $1.9 \pm 0.1$ & $1.9 \pm 0.1$ & ns \\
\hline PBF [\%] & $12.2 \pm 3.5$ & $11.1 \pm 3.4$ & ns \\
\hline $\mathrm{HR}$ [bpm] & $62.0 \pm 13.0$ & $59.0 \pm 9.0$ & 0.05 \\
\hline $\mathrm{SBP}[\mathrm{mm} \mathrm{Hg}]$ & $123.2 \pm 18.3$ & $124.6 \pm 13.6$ & ns \\
\hline $\mathrm{DBP}[\mathrm{mm} \mathrm{Hg}]$ & $81.4 \pm 11.7$ & $77.5 \pm 10.8$ & 0.05 \\
\hline LVMI $\left[\mathrm{g} / \mathrm{m}^{2}\right]$ & $102.1 \pm 16.4$ & $158.0 \pm 25.6$ & 0.000 \\
\hline LVEDD [mm] & $49.3 \pm 5.7$ & $53.9 \pm 4.0$ & 0.002 \\
\hline IVSDD [mm] & $10.0 \pm 1.7$ & $12.1 \pm 0.6$ & 0.05 \\
\hline LVPWT [mm] & $9.0 \pm 1.4$ & $11.0 \pm 1.1$ & 0.01 \\
\hline LVEF [\%] & $60.3 \pm 5.3$ & $61.0 \pm 4.2$ & ns \\
\hline $\mathrm{SV}[\mathrm{mL}]$ & $87.2 \pm 12.5$ & $94.4 \pm 18.8$ & 0.000 \\
\hline $\mathrm{RBC}\left[10^{12} / \mathrm{L}\right]$ & $5.1 \pm 0.1$ & $5.2 \pm 0.2$ & ns \\
\hline $\mathrm{HGB}[\mathrm{g} / \mathrm{dL}]$ & $15.5 \pm 2.6$ & $15.8 \pm 1.9$ & ns \\
\hline WBC $\left[10^{9} / L\right]$ & $6.6 \pm 1.1$ & $6.5 \pm 0.8$ & ns \\
\hline $\mathrm{VO}_{2 \max }[\mathrm{mL} / \mathrm{kg} / \mathrm{min}]$ & $61.5 \pm 7.9$ & $62.7 \pm 5.5$ & ns \\
\hline$P_{\max }[$ Watt $]$ & $372.0 \pm 53.4$ & $400.0 \pm 120.0$ & 0.05 \\
\hline
\end{tabular}

BMI - body mass index; BSA - body surface area; PBF - percent of body fat; HR - heart rate; bpm - beats per min; SBP - systolic blood pressure; DBP - diastolic blood pressure; LVMI - left ventricular mass index; IVSDD intraventricular septum diameter during diastole; LVPWT - left ventricular posterior wall thickness during diastole; LVEF - left ventricular ejection fraction; SV - stroke volume; RBC - red blood cells; HGB - hemoglobin; WBC - white blood cells; $\mathrm{VO}_{2 \max }$ - maximal oxygen consumption; $\mathrm{P}_{\max }$ peak aerobic power (maximal power); results were expressed as mean \pm standard deviation; $\mathrm{p}$ - significant differences between non-LVH and LVH subjects $\mathrm{p} \leq 0.05$; ns - no significant differences between non-LVH and LVH subjects $p>0.05$.

oxygen transport and distribution. In cardiovascular adaptation maximal aerobic efficacy increases by $75-80 \%$ in well-trained endurance athletes. However, LVH athletes demonstrated significantly higher $\mathrm{P}_{\max }$ compared to nonLVH athletes, we did not observe significant differences in $\mathrm{VO}_{2} \mathrm{max}$ between $\mathrm{LVH}$ and non-LVH athletes. This 
Fig. 1. Expression profiles of MYL9 and IL6ST in consecutive phases of the experiment $\left(T_{\text {rest, }}, T_{\max }, T_{15}\right)$; the mean values $(n=3)$ for cyclists with left ventricular hypertrophy (LVH) and without left ventricular hypertrophy (non-LVH)

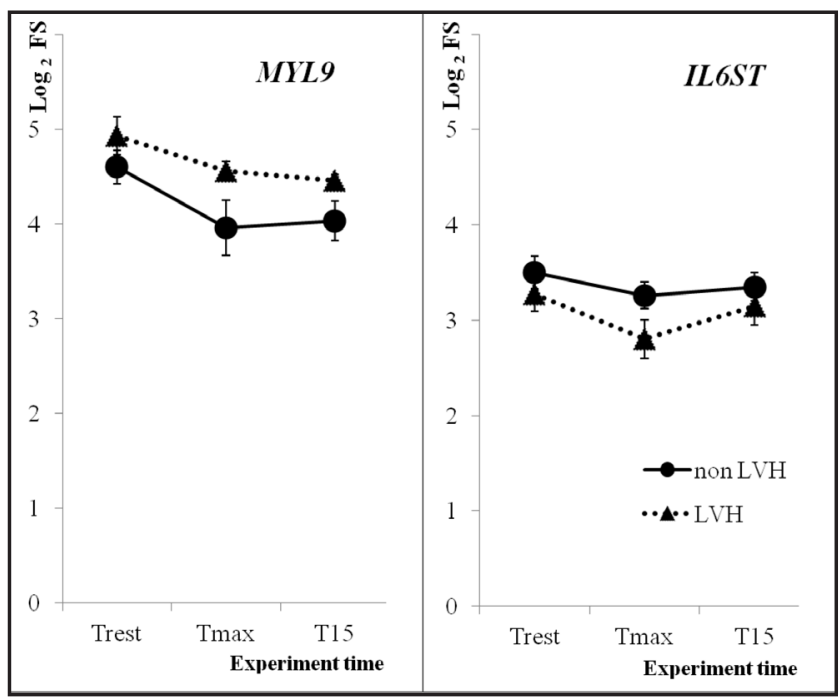

finding seems to confirm the hypothesis that aerobic performance depends on changes in the hemodynamics of the circulation system and cardiac remodeling. It has been concluded on the basis of echocardiographic parameters that the response of elite Polish road cyclists, with a similar length of training, age, body mass index, body surface area, maximal oxygen consumption and blood morphology, to regular training is not uniform. Our results demonstrated adaptive hypertrophy in the LVH subjects and normal cardiac dimensions when compared to the non-LVH group; no impairment of cardiac function was observed. ${ }^{4}$ Cardiac remodeling is more intensive in cyclists than other athletes, and presents a supernormal pattern of LV diastolic function in relation to nonathletes. ${ }^{20}$ Physiological hypertrophy is caused by a proportional increase in myocardium cell length and width. Greater body size is associated with a larger heart. In addition to LV mass, LV volumes at end-diastole and the resultant stroke volume are larger in males. In athletes who participated in endurance training (e.g. cycling), both hemodynamic overload of the heart and high left ventricle wall tension of the heart may lead to eccentric ventricular hypertrophy. Additionally, the type and intensity of the training, as well as age, influence the cardiovascular adaptation and can augment the differences in structure and function of an athlete's heart. A high prevalence of heart hypertrophy was documented in male athletes and was related to their body surface, type, and intensity of training. 6,7,19,20 The relationship between the LVDD and LVMI and age, time and training intensity depend on the sport discipline and individuality of athletes. Therefore, in order to rule out the effect of somatic parameters on metabolic variables, athletes who participated in our study had comparable baseline body weight and body surface. Moreover, the training status and regime was the same. Since the athletes' somatic characteristics and training status did not differ, we might hypothesize that the results of our study most likely reflect the exercise-induced expression of genes related to cardiac hypertrophy in the circulating blood.

Significant changes in the $\mathrm{T}_{\max }$ were observed regarding MYL9 and IL6ST. Both genes play a crucial role in the physiology and pathology of the cardiovascular system. ${ }^{10,21}$ Differential gene expression profiles in response to exercise clearly indicate that not only exercise-related but also post-exercise transcriptional changes may be of importance in adaptive cardiac hypertrophy. This finding is in agreement with those of Gielen et al. ${ }^{9}$, who found that molecular regulation in response to endurance exercise differed in athletes with heart hypertrophy, and that these differences might result in greater improvement in cardiac function.

To our knowledge, this is the first analysis of the expression of genes related to cardiac hypertrophy in cyclists, and this could help differentiate between athletes with and without this condition based on their peripheral blood. The up-regulation of MYL9 observed in the leukocytes of LVH athletes may suggest that exercise training has a beneficial effect on contractile protein content and/ or the stimulation of contractile protein isoforms. MYL9 (MLC2, MRLC1, MYRL2) is a gene encoding a muscle and actin-dependent protein called myosin regulatory light polypeptide 9.22,23 MYL9 expression was observed in the vascular tissue after injuries, depending on the age of rats used as specimens; upregulation of this gene was associated with increased vascular permeability. ${ }^{21}$ The results of in vivo and in vitro experiments on the potential signaling pathways that might regulate cardiac related genes during growth and hypertrophy are not unambiguous. ${ }^{24-26}$ Differential regulation of transcripts involved in inflammatory response, signal transduction, energy metabolism and mitochondrial function differentiate maladaptive from adaptive cardiac hypertrophy. ${ }^{14,25}$ Additionally, our findings confirm the difference between the expression profile of IL6ST in LVH and non-LVH cyclists in $\mathrm{T}_{\max }$ (Fig. 1). IL6ST encodes a transmembrane protein of the interleukin- 6 signal transducer known as glycoprotein 130 (gp130; also referred to as IL6- $\beta$ or CD130). ${ }^{26,27}$ Physiologically, this protein serves as a cardioprotective molecule against physiological stress and cardiomyocyte damage; it causes compensatory heart hypertrophy and maintains cardiac function. The study of gp130 knockout mice indicates that gp130 might play a key role in the regulation of cardiac myocyte apoptosis and in cardiomyopathy progression. ${ }^{26}$ It has also been suggested that IL6ST plays a role in the growth and survival of neonatal rat ventricular cardiomyocytes. ${ }^{28.29}$ By stimulating the secretion of circulating IL- 6 and, consequently, IL6ST, physical exercise has an anti-inflammatory effect. ${ }^{30}$ The detection of IL6ST in the peripheral blood of athletes suggests 
that the gene takes part in the body's defense against the effects of extreme physical effort.

The physiological role of genes estimated for exercise capacity of athletes and their effect on cardiac hypertrophy is not yet known. Both genes in both groups show a similar trend, although their levels in the LVH are different. An increased level of MYL9 may explain the better performance of the cyclists with LVH (higher $P_{\max }$ ), while the decreased level of IL6ST probably provides good adaptation to physical effort and a good prognosis in terms of hypertrophy. Expression of these genes might have a protective effect on the heart and are more characteristic in the circulating blood during maximum intensities of exercise.

The limitations of this study were its small group of tested cyclists and the high cost of microarrays. However, the conclusion remains valid due to the need for medical care for endurance-trained athletes, who are an increasing part of the patient population. Heart monitoring is obligatory in the prevention of the sudden death of intensively trained athletes. Searching for detecting marker genes or monitoring cardiac hypertrophy can contribute to reducing the costs of medical care. Despite the limitations in the number of athletes surveyed, studies show the importance of this medical problem (heart remodeling in response to long-term exercise training and differences in the gene expression of circulating blood in response to effort) and a possible way for it to be observed.

Intense cycling can lead to eccentric hypertrophy (LV) with differences in heart structure and function. Our studies indicate the benefits of echocardiography measurements for cyclists. Additionally, we speculate that MYL9 and IL6ST may act as important peripheral regulators of myocardial morphology and function. They can show progression in the left ventricular hypertrophy for trained athletes with similar anthropometric parameters; further multicenter studies are needed to confirm these findings.

\section{References}

1. Lavie CJ, Arena R, Swift DL, et al. Exercise and the cardiovascular system: Clinical science and cardiovascular outcomes. Circ Res. 2015;117:207-219.

2. Thiene G, Corrado D, Rigato I, Basso C. Why and how to support screening strategies to prevent sudden death in athletes. Cell Tissue Res. 2012;348:315-318.

3. Oxborough $D$, Sharma $S$, Shave $R$, et al. The right ventricle of the endurance athlete: The relationship between morphology and deformation. J Am Soc Echocardiogr. 2012;25:263-271.

4. Lang RM, Badano LP, Mor-Avi V, et al. Recommendations for cardiac chamber quantification by echocardiography in adults: An update from the American Society of Echocardiograpgy and the European Association of cardiovascular imaging. Eur Heart J Cardiovasc Imaging 2015;16:233-270.

5. Leischik R, Spelberg N. Endurance sport and cardiac injury: Prospective study of recreational ironman athletes. Int J Environ Res Public Health 2014;11:9082-9100.

6. Pelliccia A, Maron MS, Maron BJ. Assessment of left ventricular hypertrophy in a trained athlete: Differential diagnosis of physiologic athlete's heart from pathologic hypertrophy. Prog Cardiovasc. Dis 2012;54:387-396.
7. Pavlik G, Major Z, Csajagi E, Jeserich M, Kneffel Z. The athlete's heart. Part II. Influencing factors on the athlete's heart: Types of sports and age. Acta Physiol Hung. 2013;100:1-27.

8. Neri Serneri GC, Boddi M, Modesti PA, et al. Increased cardiac sympathetic activity and insulin-like growth factor-I formation are associated with physiological hypertrophy in athletes. Circulation Res. 2001;89:977-982.

9. Gielen S, Schuler G, Adams V. Cardiovascular effects of exercise training molecular mechanisms. Circulation. 2010;101:1221-1238.

10. Bernardo BC, Weeks KL, Pretorius L, McMullen JR. Molecular distinction between physiological and pathological cardiac hypertrophy: Experimental findings and therapeutic strategies. Pharmacol Ther. 2010;128:191-227.

11. Baggish AL, Wood MJ. Athlete's heart and cardiovascular care of the athlete: Scientific and clinical update. Circulation. 2011;123: 2723-2735.

12. Scott JM, Warburton DE. Mechanisms underpinning exerciseinduced changes in left ventricular function. Med Sci Sports Exerc. 2008;40:1400-1407.

13. Booth FW, Chakravarthy MV, Spangenburg EE. Exercise and gene expression: Physiological regulation of the human genome through physical activity. J Physiol. 2002;543:399-411.

14. Strøm CC, Aplin M, Ploug T, et al. Expression profiling reveals differences in metabolic gene expression between exercise-induced cardiac effects and maladaptive cardiac hypertrophy. FEBS J. 2005;272:2684-2695.

15. Devereux RB, Alonso R, Lutas R, et al. Echocardiographic assessment of left ventricular hypertrophy: Comparison to necropsy findings. Am J Cardiol. 1986;57:450-458.

16. Choe SE, Boutros M, Michelson AM, Church GM, Halfon MS. Preferred analysis methods for Affymetrix GeneChips revealed by a wholly defined control dataset. Genome Biol. 2005;6:R16.

17. D'Andrea A, Caso P, Scarafile R, et al. Biventricular myocardial adaptation to different training protocols in competitive master athletes. Int J Cardiol. 2007;115:342-349.

18. Shapiro LM. Physiological left ventricular hypertrophy. Br Heart J. 1984;52:130-135

19. Horowitz I, Cafri C, Zeller L, Vodonos A, Perry ZH, Kobal SL. Athlete's heart in Israel: fact or fiction. Isr Med Assoc J. 2014;16:46-49.

20. Moro AS, Okoshi MP, Padovani CR, Okoshi K. Doppler echocardiography in athletes from different sports. Med Sci Monit. 2013;19:187-193.

21. Shehadeh LA, Webster KA, Hare JM, Vazquez-Padron RI. Dynamic regulation of vascular myosin light chain (MYL9) with injury and aging. PLoS One. 2011;6:e25855.

22. Jalagadugula G, Mao G, Kaur G, Goldfinger LE, Dhanasekaran DN, Rao AK. Regulation of platelet myosin light chain (MYL9) by RUNX1: Implications for thrombocytopenia and platelet dysfunction in RUNX1 haplodeficiency. Blood. 2010;116:6037-6045.

23. Kaneko-Kawano T, Takasu F, Naoki $\mathrm{H}$, et al. Dynamic regulation of myosin light chain phosphorylation by Rho-kinase. PLoS One. 2012;7:e39269.

24. Lauschke J, Maisch B. Athlete's heart or hypertrophic cardiomyopathy? Clin Res Cardiol. 2009;98:80-88.

25. lemitsu M, Maeda S, Miyauchi T, Matsuda M, Tanaka H. Gene expression profiling of exercise-induced cardiac hypertrophy in rats. Acta Physiol Scand. 2005;185:259-270.

26. Jin $\mathrm{H}$, Yang R, Li W, et al. Effects of exercise training on cardiac function, gene expression, and apoptosis in rats. Am J Physiol Heart Circ Physiol. 2000;279:H2994-H3002.

27. Hirota $\mathrm{H}$, Chen J, Betz UA, et al. Loss of a gp130 cardiac muscle cell survival pathway is a critical event in the onset of heart failure during biomechanical stress. Cell. 1999;97:189-198.

28. Fischer $\mathrm{P}$, Hilfiker-Kleiner D. Role of gp130-mediated signalling pathways in the heart and its impact on potential therapeutic aspects. Br J Pharmacol. 2008;153:S414-S427.

29. Wollert KC, Taga T, Saito M, et al. Cardiotrophin-1 activates a distinct form of cardiac muscle cell hypertrophy: Assembly of sarcomeric units in series IA p130/leukemia inhibitory factor receptor dependent pathways. J Biol Chem. 1996;271:9535-9545.

30. Petersen AM, Pedersen BK. The role of IL- 6 in mediating the antiinflammatory effects of exercise. J Physiol Pharmacol. 2006;57:43-51. 